PAPER • OPEN ACCESS

Content adaptation neural network method cause-specific the state of users

To cite this article: L V Khlivnenko et al 2020 J. Phys.: Conf. Ser. 1679032084

View the article online for updates and enhancements.

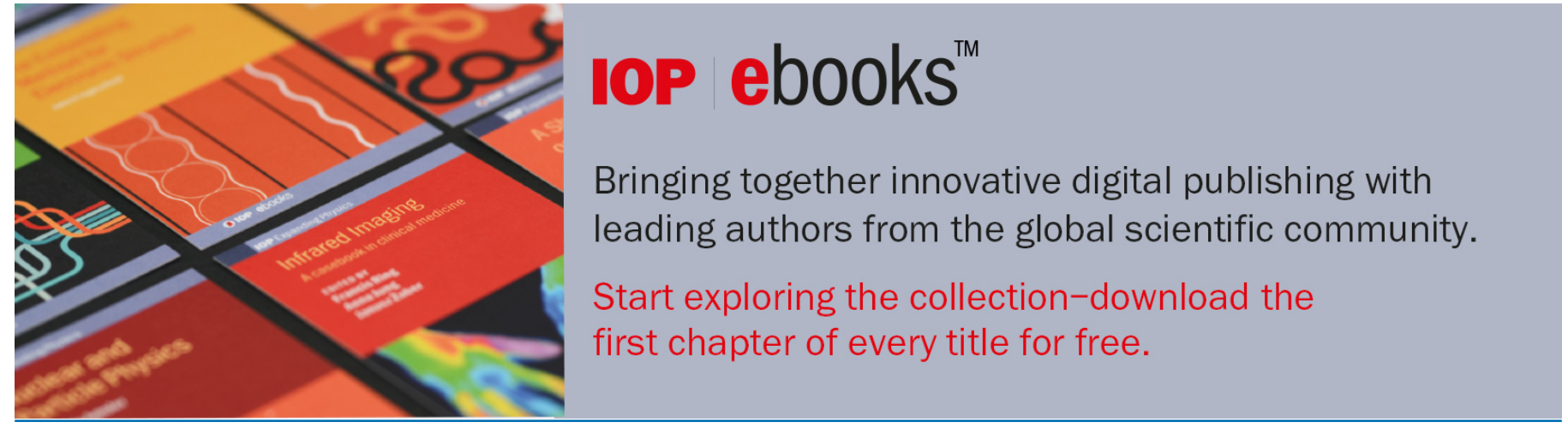

This content was downloaded from IP address 188.170 .217 .39 on $27 / 04 / 2021$ at 11:00 


\title{
Content adaptation neural network method cause-specific the state of users
}

\author{
L V Khlivnenko ${ }^{1,2}$, F A Pyatakovich ${ }^{3}$ and T I Yakunchenko ${ }^{3}$ \\ ${ }^{1}$ Department of Applied Mathematics and Mechanics, Voronezh State Technical \\ University, 14 Moskovskiy prospekt, Voronezh, 394026, Russia \\ ${ }^{2}$ MATTEST Engineering bureau of Individual Entrepreneur Eliseev Vladimir \\ Vasilyevich, 6-53 G. Perkhorovicha str., Voronezh, 394086, Russia \\ ${ }^{3}$ Department of Propedeutics of Internal Medicine and Clinical Information \\ Technologies, Belgorod State National Research University, 85 Pobeda str., Belgorod, \\ 308001, Russia
}

E-mail: khlivnenko.lv@gmail.com

\begin{abstract}
The aim of the study is to develop recommendations for adapting educational content based on the use of a neural network method for recognizing the human autonomic nervous system degree of activity. The initial data for decision making are the vectors of cardiointervals obtained with the help of the pulse sensor. The state of the autonomic nervous system is monitored by a two-layer artificial neural network of direct propagation. The artificial neural network was trained by combining gradient and stochastic training methods. A trial sample was used as a training set, consisting of 168 records of people's cardiointervals belonging to different social and age groups. The presented method for recognizing the autonomic nervous system activity degree was tested via the shared programming support environment Lazarus. As a result of network training, a set of weighting coefficient values was found, for which $100 \%$ correct recognition is performed over the entire trial sample. A personalized training system that monitors the functional state of the student varies the presentation of educational content. When the functional state changes during the training load, the virtual agent organizes the training process so as to avoid stress load.
\end{abstract}

\section{Introduction}

Currently, there is a certain increase in interest towards distance learning technologies. A well-organized information and educational environment allows you to acquire new knowledge with minimal time and financial costs. The automated process of acquiring knowledge assumes an updated format of interaction between trainers and students.

One of the main problems of this form of interaction is keeping attention to the learning process. The student must have a high level of self-discipline and ability to concentrate.

Another significant problem is the navigation in the information flow. In this regard, the learning user needs the help of a tutor in building individual educational trajectories. This function can be assumed by an intelligent agent based on a cognitive architecture.

Such a virtual agent in distance learning systems can act as a personal manager of a student, adapting educational content depending on the psychophysiological state of the student.

In this case, an important point is the indirect collection of data on the psychophysiological state [1]. 
The smart head hoop developed by BrainCo Inc. and being tested in China to monitor the attentiveness of students is unlikely to improve the comfort of the learning process. Devices, as devices operating on the basis of EEG analysis [2], are not yet very convenient in practice, since they are located on the head. Scanning brain activity through the hair often interferes with good signal readings.

Indirect data on student fatigue include eye tracking, keyboard handwriting, and heart rate variability (HRV).

HRV analysis is a non-invasive and user-friendly method of interacting with the training system [3]. A student would rather put on a fashionable fitness bracelet than a "hat" made of electrodes.

The purpose of this study is to develop recommendations for adapting educational content based on the use of a neural network method for recognizing the degree of activity of the human autonomic nervous system.

The autonomous (autonomic) nervous system (ANS) controls all vital functions of the body without the direct participation of the human consciousness (will). The ANS consists of two sections: sympathetic and parasympathetic.

The sympathetic nervous system (SNS) is responsible for mobilizing the body's resources and is activated in stressful situations. The parasympathetic nervous system (PSNS), on the other hand, is responsible for relaxation and is activated when resting.

There are six known degrees of human ANS activity: a sharp pronounced predominance of the SNS, a pronounced predominance of the SNS, a moderate predominance of the SNS, an equilibrium state between the sympathetic and parasympathetic parts of the ANS, a moderate predominance of the PSNS, a pronounced predominance of the PSNS [4].

\section{Experimental Section}

\subsection{Model inputs}

The initial data for decision-making are the vectors of cardiointervals $R R_{i}, i=\overline{1,500}$, obtained using a photoplethysmographic pulse sensor. In our case, $R R_{i}$ - is the time in seconds between high-amplitude plethysmogram waves. The current activity of the SNS and PSNS is directly related to the response of the multicircuit and multilevel circulatory regulation system [5].

The remainder $\left(R R_{i+1}-R R_{i}\right)$ is called "correction". All observed values of corrections in humans fall within the interval $(-1,55 ; 1,55)$. The static series is divided into equal sections with a length of $0.05 \mathrm{sec}$. It turns out the 61 st class of differential histogram of the distribution of the microstructure of the heart rate variability pattern, including the norm, tachy- and bradyrhythmia.

The model inputs $x_{j}, j=1,61$, are the frequencies of the obtained interval static series. The coordinates of the input vector $X=\left\{x_{i}\right\}$ are subjected to the "normalization" procedure by subtracting their sample mean and normalizing to the corrected sample mean square deviation.

\subsection{Functioning of an artificial neural network}

Monitoring of the ANS state is carried out by a two-layer artificial neural network (ANN) of direct propagation, consisting of 61 inputs $\left(x_{j}\right), 10$ neurons of the hidden layer $\left(y_{k}^{c}, k=1,10\right)$ and 6 neurons of the output layer $\left(y_{i}, i=1,6\right)$.

ANN functioning is described by formulas (1) - (2).

$$
y_{k}^{c}=f\left(\sum_{j=1}^{61} w_{k j} x_{j}\right),
$$

where $w_{k j}$ - is the weighting coefficient of the connection between the $j$-th input and $k$-th neuron of the hidden layer. Here and below, the hyperbolic tangent is chosen as the activation function: $f(s)=\left(e^{s}-e^{-s}\right) /\left(e^{s}+e^{-s}\right)$. 


$$
y_{i}=f\left(\sum_{k=1}^{10} v_{i k} y_{k}^{c}\right)
$$

where $v_{i k}$ - is the weighting coefficient of the connection between the $k$-th neuron of the hidden layer and $i$-th neuron of the output layer.

The maximum calculated value $y_{i}$ determines the class number $i$, to which the ANN classifies the input pattern of cardiointervals. The class number corresponds to one of the six conditions of the ANS.

The sets of weighting coefficients $\left\{w_{k j}\right\},\left\{v_{i k}\right\}$, used in the diagnostic mode are obtained as a result of preliminary training of the ANN on a training set classified by an expert [6].

\subsection{Artificial neural network training}

For research purposes, a test sample was used as a training set, consisting of 168 records of cardiointervals of people belonging to different social and age groups [7].

In order to train the ANN, the expert doctor, analyzing the shape of the scattergram built according to the patterns of cardiointervals from the training set, distributed all the examples into 6 target classes. In the target output vectors, the position $t_{i}$ of the "correct" class was marked with a value of 0.5 . The rest of the coordinates took on the values: -0.5 .

ANN training was carried out by combining gradient and stochastic training methods.

The weight coefficients were adjusted according to formulas (3) - (4).

$$
\begin{gathered}
v_{i k}^{N+1}=v_{i k}^{N}-\eta \alpha\left(\delta_{i}\right)_{l}^{N}\left(y_{k}^{c}\right)_{l}^{N}+(1-\eta) v_{c}, \\
w_{k j}^{N+1}=w_{k j}^{N}-\eta \alpha\left(\sum_{i=1}^{6}\left(\delta_{i}\right)_{l}^{N} v_{i k}^{N}\right)\left(1-\left(\left(y_{k}^{c}\right)_{l}^{N}\right)^{2}\right)\left(x_{j}\right)_{l}^{N}+(1-\eta) w_{c},
\end{gathered}
$$

where $N$ is a discrete moment in time, $\eta$ - is a coefficient that controls the relative values of the gradient and stochastic components of the weight, $\alpha$ is the learning rate, $\delta_{i}=\left(y_{i}-t_{i}\right)\left(1-y_{i}^{2}\right), v_{c}$ and $w_{c}$ are stochastic corrections of the corresponding weights determined by formula (5).

$$
v_{c}=w_{c}=\alpha T(N) \operatorname{tg}(P(w)),
$$

where $T(N)$ is the artificial temperature, considered as a function of time:

$$
T(N)=\frac{T_{0}}{1+N}
$$

$P(w)$ is a random number from a uniform distribution over the interval $(-\pi / 2 ; \pi / 2)$.

The negative part was introduced for random determination of the sign of the correction.

After adjusting the weighting coefficients, the value of the objective function determined by formula (7) was recalculated.

$$
E(v(N), w(N))=\left(\sum_{i=1}^{6}\left(f\left(\sum_{k=1}^{10} v_{i k}(N) f\left(\sum_{j=1}^{61} w_{k j}(N) x_{j}^{(N)}\right)\right)-t_{i}^{(N)}\right)^{2}\right) / 2
$$

If the network error decreased, then such weighting changes were retained. To prevent the network learning process from getting stuck in the local minima of the objective function, but moving in the direction of searching for a global minimum, with an increase in the network error, new weight coefficients were preserved with a probability determined by the Cauchy distribution:

$$
p(w)=\frac{T(N)}{T^{2}(N)+w^{2}},
$$


where $w$ is the stochastic weight correction.

Integrating $p(w)(8)$ from 0 to $w$ and solving the resulting equation with respect to $w$, we have formula (5), where $P(w)=\int_{0}^{w} p(w) d w$.

When initializing the network, all weight coefficients took random values from the segment $[-0,3 ; 0,3]$. Learning speed $[-0,3 ; 0,3]$.

\subsection{Model trialability}

The presented method for recognizing the degree of ANS activity was tested in the free Lazarus programming environment. The authors have developed a fully functional application that allows you to form random subsamples from the available data set, train a neural network, and test the effectiveness of training on control subsets [8].

As a result of multiple network training, a set of weight coefficient values was found, for which $100 \%$ correct recognition is performed over the entire test sample.

\section{Results and discussion}

The presented neural network method for recognizing ANS activity based on HRV analysis allows monitoring the functional state of a person in real time in a format that is quite comfortable for him.

A personalized training system (virtual agent), "observing" changes in the ANS activity, varies the presentation of educational content.

At rest, in younger people, there is an equilibrium state between the sympathetic and parasympathetic divisions of the ANS. Moderate predominance of SNS and PSNS characterizes the individual characteristics of the organism. An important point is the drift of the functional state during the learning load, which can be considered a stress factor.

There are three stages of stress [9]. At the first stage of anxiety or the stage of emergency mobilization, the SNS is activated. The second stage of resistance or the stage of stable adaptation involves moderate fluctuations between the activation of SNS and PSNS. The third stage of exhaustion arises as a response to super-strong or super-prolonged loads and is accompanied by extreme states: a pronounced predominance of the SNS in the case of emotional irritation or a pronounced predominance of the PSN in a state of emotional stupor.

Understanding of physiological processes allows you to "defuse" the situation in advance of the onset of fatigue and rejection of the learning process by changing the tactics of learning.

In the case of the start of a training session with a predominance of pronounced and sharply expressed activity of the SNS, the training system offers relaxing modules (play, light tasks for repetition, communication) until the equilibrium state of the ANS is stabilized or one of the components is moderately dominant. It is within these boundaries that the delivery of the main educational content is realized.

If initially the student is strongly relaxed with a manifested predominance of PSNS activity, he is offered tonic modules (game, test, and mentor) until the functional state stabilizes. If this does not happen, then the training session is postponed to another time. The training system acts in the same way when the functional state approaches a critical one (pronounced predominance of SNS or PSNS activity).

\section{References}

[1] Pyatakovich F A, Khlivnenko L V, Yakunchenko T I, Makkonen K F and Mevsha O V 2018 J. Allergology and Immunology 19(01) 58-9

[2] Shishkin S L 2019 Proc. of $5^{\text {th }}$ Int. Conf. BCI: Science and Practice (Samara, RF) 3-4

[3] Pytakovich F A, Khlivnenko L V, Yakunchenko T I, Makkonen K F and Mevsha O V 2018 Journal of Advanced Research in Dynamical and Control Systems (JARDCS) 10-Special Issue 
1853-7

[4] Makkonen K F and Pyatakovich F A 2009 International journal of applied and fundamental research 2 17-20

[5] Baevsky R M et al. 2001 J. Bulletin of Arrhythmology 24 65-87

[6] Pyatakovich F A, Khlivnenko L V, Mevsha O V, Yakunchenko T I and Makkonen K F 2018 J. Modern issues of biomedicine 2(01) 171-83

[7] Khlivnenko L V, Pytakovich F A, Vasiliev V V and Yakunchenko T I 2015 J. System analysis and management in biomedical systems 14(03) 544-8

[8] Pytakovich F A, Khlivnenko L V, Yakunchenko T I, Makkonen K F and Mevsha O V 2017 Indo American Journal of Pharmaceutical Sciences (IAJPS) 4(09) 3075-9

[9] Lapkin M M and Trutneva E A 2018 Selected lectures on the normal physiology (Moscow: GEOTAR-Media) p 237 